\title{
Manejo perioperatorio del paciente neuroquirúrgico con COVID-19
}

Perioperative management of the neurosurgical patient COVID-19

\author{
Dra. María Areli Osorio-Santiago,* Dra. Luisa Piedad Manrique-Carmona ${ }^{\ddagger}$
}

RESUMEN. La enfermedad provocada por el virus COVID-19 causa el síndrome infeccioso respiratorio agudo severo conocido como SARS-2; este virus puede penetrar el sistema nervioso central y afectar neuronas y células gliales. Clínicamente se manifiesta como encefalitis, ictus isquémico e incluso polineuropatía. Cuando nos enfrentamos a un paciente neuroquirúrgico COVID-19 positivo se requiere una evaluación rápida pero detallada del estado general y neurológico del enfermo. Toda cirugía en presencia de un paciente COVID-19 positivo deberá realizarse con medidas de protección de nivel alto durante la cirugía, el uso de craneotómos y electrocauterios deberá minimizarse, a fin de reducir la producción de aerosol. Los procedimientos transesfenoidales endonasales deben ser evitados durante el período de la pandemia. Nos basamos en el consenso de expertos realizados por la SNACC (Society for Neuroscience in Anesthesiology and Critical Care) para emitir recomendaciones adaptadas a nuestro entorno. La terapia endovascular es una alternativa viable a la trombólisis intravenosa para el reestablecimiento de la circulación. Se ha observado mejor reperfusión en aquellos pacientes que no recibieron sedación o ésta fue ligera, comparado con los pacientes que recibieron anestesia general. En algunos casos no es indispensable la extubación postquirúrgica (especialmente en pacientes infectados con COVID-19), por lo que se deberá mantener sedación profunda y trasladar al paciente a la Unidad de Cuidados Intensivos (UCI). Es importante recordar que la infección por COVID-19 se ha evidenciado en los principales sistemas, causando daño multiorgánico en los pacientes susceptibles, razón por la cual se deberá vigilar estrechamente todos los datos que nos lleven a pensar en disfunción a corto y mediano plazo. La toma de decisiones éticas respecto a qué paciente es candidato a una craniectomía descompresiva urgente o tratamiento endovascular, si más de un vaso está ocluido, se deberá discutir en conjunto con el equipo tratante para no caer en ensañamiento terapéutico, o bien, en la omisión de un tratamiento oportuno.

ABSTRACT. The disease made by the COVID-19 virus causes the infectious respiratory acute severe syndrome known as SARS-2, this virus can penetrate the central nervous system affecting neurons and glial cells, is clinically manifested as encephalitis, ischemic stroke, and even polyneuropathy. When we confront a neuroquirurgic patient with positive COVID-19 we require a fast but detailed evaluation of the general and neurological status of the sick. Every surgery in presence of a positive COVID-19 patient, must be realized with high-level protection measures while the surgery happens. The use of craniotomes and electrocautery should be minimized in order to reduce aerosol production. Transsphenoidal endonasal procedures should be avoided during the period of the pandemic. We're based in the concense of experts made by the SNACC (Society for Neuroscience in Anesthesiology and Critical Care) to emit recommendations adapted to our environment. Endovascular therapy is a viable alternative to the intravenous trombolisis for the reestablishment of circulation. It has been observed better reperfusion in those patients who didn't received sedation, or this was light compared with the ones who did received general anesthesia. In some cases it's indispensable the post-surgical extubation (specially in those patients who're infected with COVID-19), so it should be kept as deep sedation and should traslade the patient to UCI. Is important the remember that infection by COVID-19 has been shown in the principal systems, causing multi-organ damage in susceptible patients, reason of why we'll have to look tightly after every data that takes us to think in dysfunction in small and medium term. Ethical decision making regarding which patient is a candidate to a urgent decompressive craniectomy or endovascular treatment, if more than a vase is occluded, this will have to be discussed with the treating team to not fall in therapeutic cruelty or well in the omission of a opportune treatment.

\section{INTRODUCCIÓN}

$\mathrm{H}_{1}$ ablaremos de aquellos pacientes infectados por COVID-19 que durante la evolución de la enfermedad presentan afección al Sistema Nervioso Central. Nos referiremos a aquellos padecimientos o complicaciones que requieran tratamiento urgente como craniectomía descompresiva (por edema cerebral secundario a encefalitis, o bien, derivado de un territorio
Anestesiología

Octubre-Diciembre 2020

Vol. 43. No. 4. pp 245-250

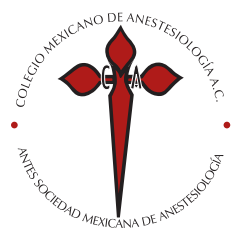

Palabras clave: COVID-19, neuroquirúrgico, manejo perioperatorio, craneotomía, terapia endovascular.

Keywords:

COVID-19, neurosurgical, perioperative management, craneotomy, endovascular therapy.

* Médico Anestesiólogo, Sociedad de Médicos Fundación Clínica Médica Sur. ‡ Profesora Titular del Curso de Neuroanestesiología Instituto Nacional de Neurología y Neurocirugía.

Solicitud de sobretiros:

Dra. María Areli Osorio-Santiago E-mail:

grupodetrabajoneuroanestesia@ gmail.com

Recibido para publicación: 08-07-2020

Aceptado para publicación: 29-07-2020 
isquémico), o manejo endovascular para reestablecer la circulación sanguínea en la vasculatura cerebral. Este artículo está encaminado a realizar recomendaciones que se adapten a nuestros recursos y a limitar el contagio entre el personal de salud y otros pacientes. Es vital estar preparados y, en lo posible, planear con cuidado cada una de las etapas.

La enfermedad provocada por el virus COVID-19 causa el síndrome infeccioso respiratorio agudo severo, conocido como SARS-2, este virus no siempre permanece confinado al tracto respiratorio, en poblaciones vulnerables ${ }^{(1)}$ puede penetrar al sistema nervioso central y afectar neuronas y células gliales. Clínicamente se manifiesta como encefalitis, ictus isquémico e, incluso, polineuropatía ${ }^{(2)}$. La asociación con eventos trombóticos arteriales y venosos del sistema nervioso se ha reportado hasta $36 \%$ en 214 pacientes en la ciudad epicentro de la pandemia (Wuhan, China) ${ }^{(3)}$.

Si bien a nivel mundial existe disminución importante en los casos admitidos por ictus vascular, se ha identificado que la infección por COVID puede estar relacionada con eventos cerebrovasculares en sus formas moderada y severa, aunque esta relación aún no está bien determinada, se sabe que esta enfermedad puede presentar síntomas neurológicos, de éstos, los más frecuentes son: convulsiones (en pacientes con encefalitis por COVID-19), mareo $16.3 \%$, cefalea $13.1 \%$, anosmia/disgeusia 5.8\% y accidente isquémico vascular 2.8$5.7 \%{ }^{(4)}$. Diversos mecanismos fisiopatológicos de la infección por COVID-19 han sido identificados como posibilidad para incremento de riesgo de ictus isquémico vascular, sin haber sido completamente aprobados hasta el momento, estos mecanismos incluyen: estado de hipercoagulabilidad, que la evidencia que se ha planteado es el incremento de los niveles de dímero $\mathrm{D}$ y una respuesta inflamatoria exagerada conocida como «tormenta de citoquinas», esto se ha considerado marcador de la forma severa de la enfermedad ${ }^{(5)}$.

La invasión directa del coronavirus al sistema nervioso central se ha descrito como encefalopatía aguda necrosante ${ }^{(4)}$.

El SARS-CoV-2 fue aislado de líquido cefalorraquídeo por secuenciación de genes (secuencias de ácido ribonucleico detectadas HCoV-OC43 y HCoV-229E), lo cual confirma su capacidad para atravesar la barrera hematoencefálica ${ }^{(6)}$.

\section{PERÍODO PREANESTÉSICO}

Cuando nos enfrentamos a un paciente neuroquirúrgico COVID-19 positivo, se requiere una evaluación rápida pero detallada del estado general y neurológico del enfermo.

Los pacientes asintomáticos pueden esparcir virus( ${ }^{(7)}$. Así que todos los que serán llevados a cirugía deben ser rastreados para COVID-19, incluyendo la medición de temperatura, interrogar síntomas, prueba de anticuerpos y TAC de tórax ${ }^{(8)}$.

En relación con la terapia endovascular, la literatura es escueta, en cuanto a la evaluación y preparación preanestésica ${ }^{(9)}$, de tal manera que al realizar un procedimiento de esta naturaleza, debemos evaluar al paciente como se describe en la Tabla 1.

El trabajo en equipo es fundamental en el abordaje paso a paso del paciente neuroquirúrgico con COVID-19 positivo, tanto la valoración prequirúrgica como la preanestésica estarán encaminadas a detectar cada uno de los aspectos que se deben abordar de manera precisa, a fin de asegurar un proceso fluido y expedito, con el mínimo de interrupciones para evitar la exposición al máximo (Tabla 1).

\section{PERÍODO TRANSANESTÉSICO}

Por la naturaleza de la evolución del edema cerebral y/o ictus isquémico vascular, todos los pacientes que ingresen a procedimientos anestésicos serán considerados urgencias; sin omitir las recomendaciones de seguridad, protección personal y precauciones que se deben tomar para evitar la infección por COVID-19 ${ }^{(10)}$.

Aun cuando los estudios clínicos son limitados y diversas publicaciones son experiencias y opiniones respecto al manejo transanestésico de paciente neuroquirúrgico infectado

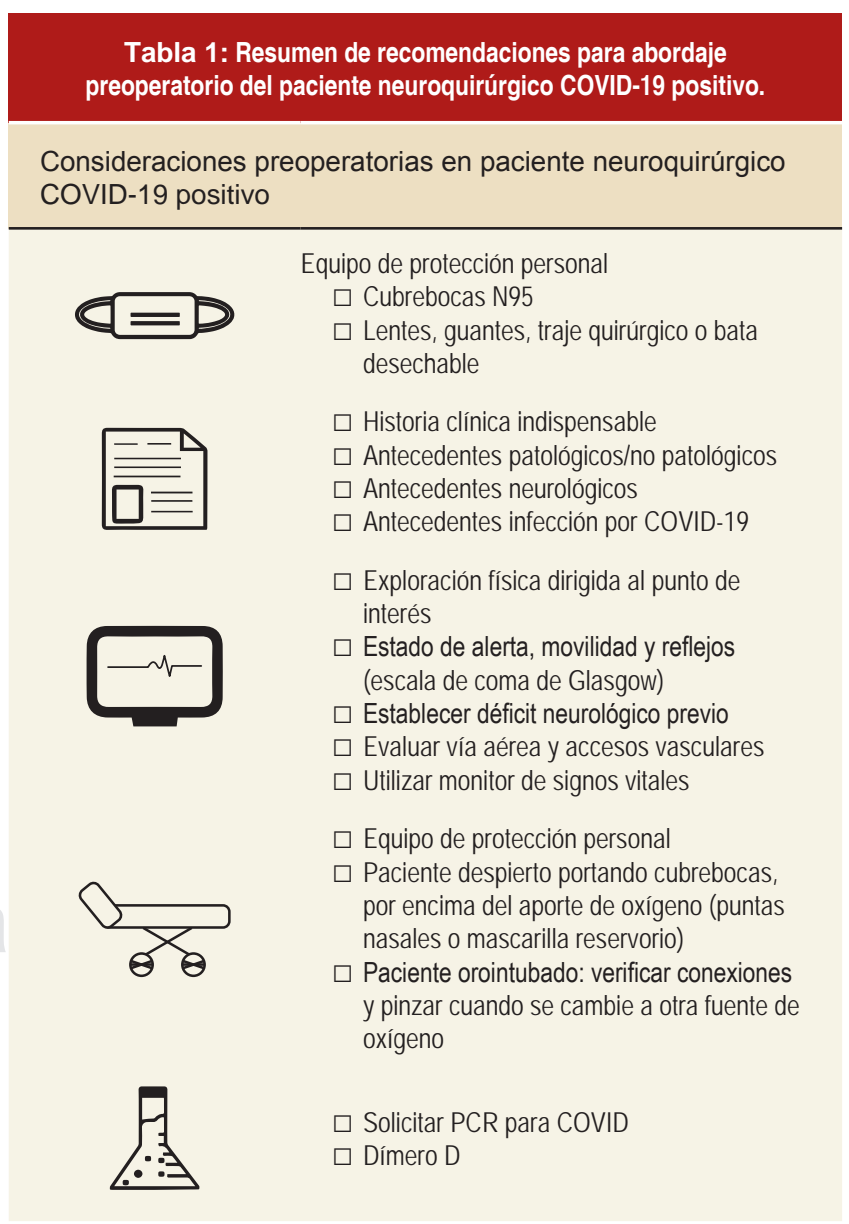


por COVID, realizamos una revisión exhaustiva de todos los parámetros que nos ocupan, en cirugía neurológica y terapia endovascular.

\section{PROCEDIMIENTOS QUIRÚRGICOS}

Al igual que el ataque isquémico agudo, las encefalitis de cualquier origen ocasionalmente pueden cursar con incremento importante de la presión intracraneal, acompañado de deterioro neurológico, una vez que las medidas conservadoras (soluciones hipertónicas, corticosteroides, hiperventilación, hipotermia, barbitúricos, agentes osmóticos) han fracasado, se puede optar como última oportunidad por la craniectomía descompresiva ${ }^{(6)}$.

Un metaanálisis que incluyó 1,800 pacientes COVID-19 positivo, reveló que la cuenta plaquetaria está asociada con la severidad. La trombocitopenia puede dar lugar a resangrado que resulte en desenlace poco favorable ${ }^{(11)}$.

No se recomienda el uso de esteroides de manera rutinaria, ya que éstos pueden exacerbar el daño pulmonar ${ }^{(12)}$.

Aún no hay indicaciones específicas en relación con los injertos craneales ${ }^{(6)}$.

Toda cirugía, en presencia de un paciente COVID-19 positivo, deberá realizarse con medidas de protección de nivel alto ${ }^{(13)}$; durante la cirugía, el uso de craneótomos y electrocauterios deberá minimizarse, a fin de reducir la producción de aerosol ${ }^{(14)}$.

Se debe usar una sala de quirófano con presión atmosférica negativa y acceso independiente para todos los casos sospechosos y confirmados de COVID-19 positivo $^{(15)}$.

En lo posible, sólo un neurocirujano experimentado debe realizar los procedimientos, a fin de reducir el tiempo operatorio y prevenir la exposición a otros médicos ${ }^{(16)}$.

Se debe tener especial precaución durante la cirugía de base de cráneo anterior para evitar la apertura de los senos frontal o etmoidal ${ }^{(17)}$.

Aunado al potencial riesgo de transmisión, el tiempo de uso del electrocauterio monopolar o bipolar debe ser reducido y su programación con mínimo poder, para disminuir la dispersión por aerosol ${ }^{(16)}$.

Los procedimientos transesfenoidales endonasales deben ser evitados durante el período de la pandemia ${ }^{(17)}$. Se recomienda también utilizar gubias y cinceles, en lugar de instrumentos eléctricos durante la exposición quirúrgica y evitar el uso de taponamiento nasal, que al removerse estimula secreciones y tos ${ }^{(18)}$.

Posterior a la extubación, se recomienda que el paciente utilice cubrebocas lo más pronto posible ${ }^{(19)}$.

\section{PROCEDIMIENTOS ENDOVASCULARES}

Si bien la literatura relacionada con el manejo anestésico en terapia endovascular durante el ataque isquémico agudo es limitada (previo a la pandemia) y no hay estudios controlados respecto a las técnicas o fármacos anestésicos empleados, debemos tratar a estos enfermos con los recursos con los que contemos en nuestra institución, adaptarlos y optimizarlos a nuestro escenario. Nos hemos apoyado en el consenso de expertos realizados por la SNACC (Society for Neuroscience in Anesthesiology and Critical Care) para emitir recomendaciones adaptadas a nuestro entorno ${ }^{(20)}$.

La terapia endovascular es una alternativa viable a la trombólisis intravenosa para el reestablecimiento de la circulación para un vaso ocluido por un trombo en pacientes seleccionados, esto en centros hospitalarios que cuentan con infraestructura adecuada ${ }^{(9)}$.

Técnica anestésica: se ha observado mejor reperfusión en aquellos pacientes que no recibieron sedación o ésta fue ligera, en comparación con quienes recibieron anestesia general. Se ha reportado un gran número de casos de rotación de técnica anestésica (sedación a general) por agitación, náusea, alteración del estado de alerta o sangrado intracraneal ${ }^{(21)}$.

Los fármacos más usados en los casos reportados son: fentanilo, midazolam, propofol, ketamina y dexmedetomidina.

El uso de anestesia local más sedación consciente durante el tratamiento endovascular se ha asociado a baja mortalidad con mejor resultado neurológico, comparado con la anestesia general. Elegir la técnica anestésica dependerá del estado neurológico del paciente $\mathrm{e}^{(22)}$.

En aquéllos con adecuado estado neurológico preprocedimiento, se prefiere realizar con enfermo despierto o sedación ligera. Elegir la técnica anestésica dependerá del estado neurológico del paciente. No se hace una recomendación para sedación o anestesia general con fármacos específicos. El anestesiólogo decidirá cuáles emplear, así como el nivel de sedación de acuerdo al estado del paciente.

La técnica anestésica no influye en la presencia o no de complicaciones atribuibles al microcatéter o la microguía como perforaciones o sangrado intracraneal ${ }^{(23)}$.

Siempre estar preparado para convertir la sedación en anestesia general.

Oxigenación y ventilación: la hipoxia tiene efectos devastadores en el resultado neurológico posterior al ataque isquémico ${ }^{(24)}$. Estos pacientes si no están intubados, pueden causar hipoxemia por alteración central de la respiración, apnea del sueño, debilidad de los músculos de la respiración. La hiperoxia se ha propuesto como medida neuroprotectora para preservar tejido y extender la ventana terapéutica para la administración de fármacos trombolíticos; sin embargo, no ha demostrado beneficios en cuanto a supervivencia o recuperación neurológica ${ }^{(25)}$.

En pacientes orointubados con compromiso pulmonar se deberá continuar el protocolo de ventilación empleado en la Unidad de Terapia Intensiva (UTI). No hay datos relacionados con el uso de hipercapnia para promover vasodilatación cerebral regional $^{(26)}$. 
No hay datos específicos de los niveles de $\mathrm{CO}_{2}$ espirado que se deben mantener durante el tratamiento endovascular, el uso de hipocapnia como medida terapéutica para redistribuir el flujo sanguíneo cerebral durante isquemia focal.

La hipocapnia se ha relacionado con un pobre pronóstico durante isquemia cerebral aguda ${ }^{(27)}$.

En los casos de pacientes orointubados procedentes de UTI se deberá continuar con el protocolo de ventilación. Se mantendrá con suplemento de oxígeno y vigilancia a aquéllos que presenten adecuado estado de alerta y patrón ventilatorio. Se debe monitorizar de manera intermitente la $\mathrm{PaO}_{2}$ y la $\mathrm{PaCO}_{2}$ con toma de muestras desde el acceso endovascular. Se recomienda titular la $\mathrm{FiO}_{2}$ para mantener $\mathrm{SPO}_{2}>92 \%$. Mantener en medida de lo posible normocapnia, evitar hipo- e hipercapnia.

Manejo hemodinámico: el decremento o incremento importante en la tensión arterial previo al tratamiento endovascular es de mal pronóstico ${ }^{(28)}$. Las cifras de tensión arterial durante el tratamiento endovascular no han sido bien determinadas.
Se debe evitar alteraciones súbitas en la presión arterial (hipo- e hipertensión). Se debe monitorizar la presión arterial al menos cada tres minutos, desde que se diagnostica el ataque isquémico. Se recomienda mantener la presión sistólica $>140$ mmHg con líquidos y vasopresores y < 180 mmHg con o sin activador tisular de plasminógeno (RTPA). Mantener presión diastólica < $105 \mathrm{mmHg}$. En caso de hipotensión, buscar la causa y tratar de manera inmediata.

Mantener estrecha comunicación con el equipo intervencionista al iniciar la reperfusión del vaso ocluido por el riesgo de sangrado, ya que éste carece de autorregulación.

Manejo de líquidos: la hipervolemia no disminuye la gravedad del caso, pero sí puede disminuir la capacidad de transporte de $\mathrm{O}_{2}$. La euvolemia puede reducir la incidencia de hipotensión después del ataque isquémico. No emplear soluciones glucosadas, sólo administrar en caso de niveles séricos de glucosa $<50 \mathrm{mg} / \mathrm{dL}$. La recomendación emitida por los expertos es mantener euvolemia durante el tratamiento endovascular ${ }^{(29)}$.

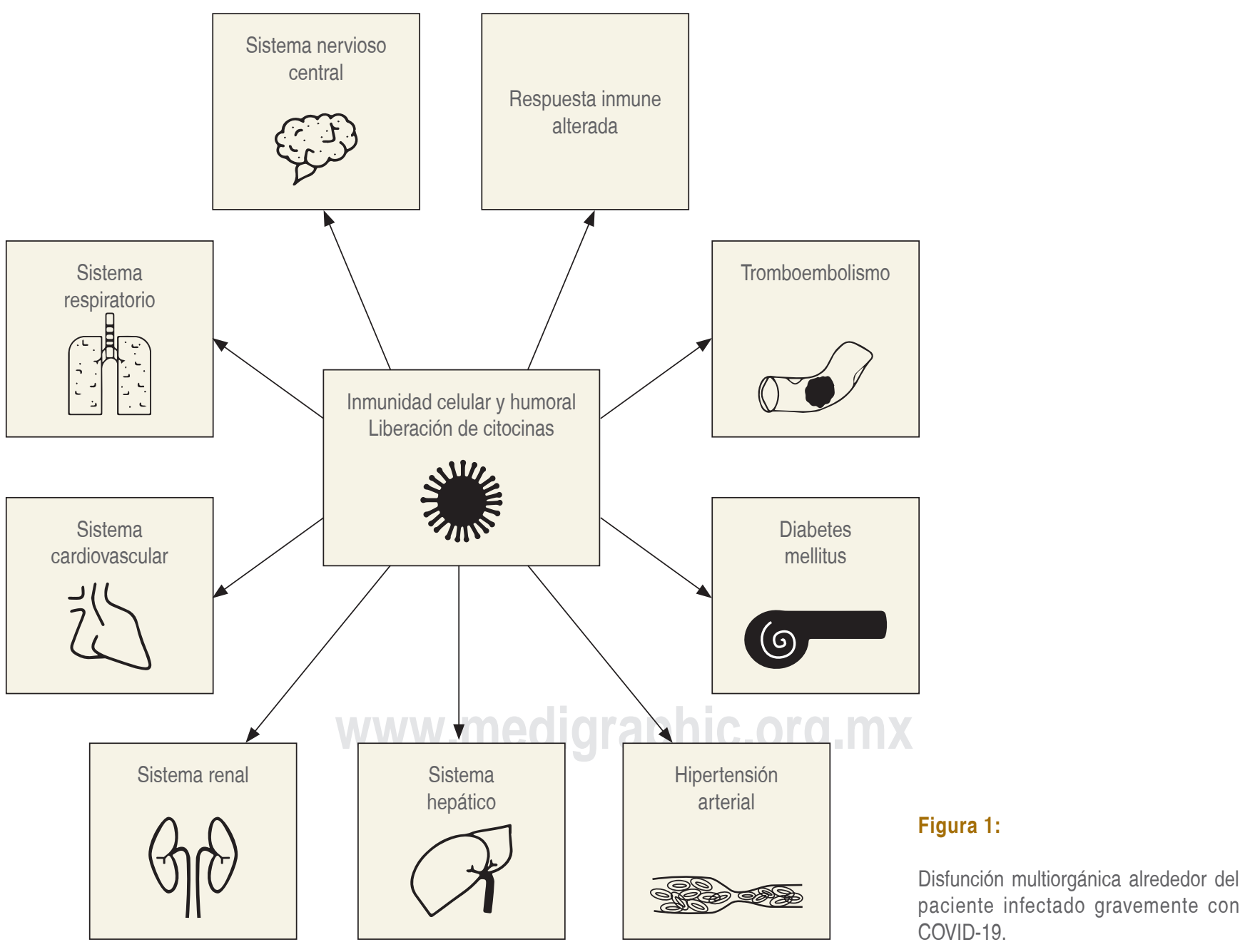


Temperatura: aproximadamente un tercio de los pacientes con ictus vascular tienen fiebre, la cual se asocia a un pobre resultado neurológico. La evidencia en relación con la hipotermia como medida de neuroprotección en pacientes con ataque isquémico agudo no existe. Se recomienda mantener entre $35-37^{\circ}$ durante el tratamiento. En caso de fiebre se deberá tratar con antipiréticos o dispositivos para disminuir la temperatura ${ }^{(30)}$. Si se presenta temblor se recomienda tratar con meperidina.

Anticoagulación: durante el tratamiento endovascular, es frecuente que se use heparina o antiagregantes plaquetarios para disminuir la incidencia de eventos trombóticos y embólicos relacionados con el uso de catéteres y stents. Debemos estar preparados para administrar heparina o protamina en caso de ser necesaria la reversión de la misma, si el infarto se convirtiera en hemorrágico.

Glicemia: la presencia de hiperglicemia es común en pacientes con ataque isquémico agudo, ésta predice un infarto mayor, pobre resultado neurológico y riesgo de mortalidad mayor, en especial en pacientes con infartos corticales ${ }^{(31)}$. Existe una asociación entre hiperglicemia y un pobre resultado clínico en pacientes tratados con terapia trombolítica que en aquéllos no tratados con RTPA. La mayoría de los datos publicados asocian niveles de glucosa $>140 \mathrm{mg} / \mathrm{dL}$ con un incremento en la extensión del infarto. La terapia intensiva con insulina puede provocar hipoglicemia, la cual se ha asociado a un resultado neurológico pobre. Se recomienda tratar la hiperglicemia con insulina en presencia de cifras séricas de glucosa de 140-185 mg/dL ${ }^{(32)}$. Se recomienda monitorizar de forma horaria la glucosa en el período transanestésico por las variaciones que puede haber bajo anestesia. La recomendación para corrección de hipoglicemia es: 500 $\mathrm{mL}$ de dextrosa al 10\% + $20 \mathrm{mmol}$ de cloruro de potasio con insulina 16 UI para conseguir el objetivo de glucosa ${ }^{(33)}$.

\section{MANEJO DE COMPLICACIONES}

El tratamiento trombolítico intravenoso o intraarterial con RTPA y la trombectomía mecánica endovascular son tratamientos efectivos; sin embargo, este último es más susceptible de complicaciones mecánicas iatrogénicas por lesión directa al vaso derivadas de microcatéteres, microguías o los dispositivos mecánicos usados para la trombectomía, convirtiendo el ataque isquémico en hemorrágico, seguido de incremento súbito de la presión intracraneal, lo cual requerirá de manera urgente tratamiento. En el caso de hemorragia subaracnoidea con hipertensión intracraneal sintomática, se deberá realizar ventriculostomía para control de la presión intracraneal; los pacientes que recibieron RTPA requerirán transfusión de plasma fresco congelado, crioprecipitados y plaquetas; asociado a estas complicaciones se deberá pensar en lesiones como disección del vaso o vasoespasmo, hematomas en sitio de punción, isquemia de la extremidad de acceso vascular y hematomas retroperitoneales ${ }^{(34-36)}$.

\section{POSTANESTÉSICO}

En algunos casos, no es indispensable la extubación postquirúrgica (especialmente en pacientes infectados con COVID-19), por lo que se deberá mantener sedación profunda y trasladar al paciente a UCI, además de aislamiento estricto y protección igual a la del traslado preoperatorio ${ }^{(37)}$.

Se discutirá concienzudamente con el equipo quirúrgico o intervencionista la conducta a seguir en el postoperatorio, sobre todo la referente al manejo de la vía aérea. Extubar o mantener intubado a un paciente dependerá de las condiciones no sólo neurológicas, sino también respiratorias; aún contamos con poca evidencia para apoyarnos y hacer recomendaciones. Pero es adecuado planear el protocolo de extubación previo al ingreso a sala de intervención ${ }^{(38)}$.

Tomar en cuenta la evolución respiratoria del paciente orientará para tomar decisiones; es indispensable una buena oxigenación para evitar complicaciones posteriores como retención de $\mathrm{CO}_{2}$ con subsecuente aumento de la presión intracraneal, neumoencéfalo, pérdida del estado de alerta, etcétera. La tos no es favorable para el aumento de la presión intracraneal, puesto que en un episodio puede alcanzar límites muy altos que no toleraría un paciente de este tipo.

Es importante recordar que la infección por COVID-19 se ha evidenciado en los principales sistemas, causando daño multiorgánico en los pacientes susceptibles, razón por la cual se deberá vigilar estrechamente todos los datos que nos lleven a pensar en disfunción a corto y mediano plazo ${ }^{(39)}$.

\section{CONCLUSIONES}

La historia sobre la pandemia SARS-CoV-2 se escribe diariamente, cada segundo cambian las cifras relacionadas con la morbimortalidad vinculada al virus y aunque se acumula evidencia día con día, aún la comunidad médica se encuentra lejos de conocer la historia natural de la enfermedad, implicaciones a largo plazo, tratamiento, pronóstico e inmunidad.

Aunque cada padecimiento tiene sus propias consideraciones, en especial el paciente neuroquirúrgico de urgencia es un desafío: «tiempo es cerebro» y es nuestro deber como Anestesiólogos garantizar procedimientos seguros en tiempo récord; adicionar el hecho de la coexistencia de SARS-CoV-2 nos enfrenta a un reto mucho mayor que implica no sólo la toma de decisiones transanestésicas, sino éticas respecto a qué paciente es candidato a una craniectomía descompresiva urgente o a tratamiento endovascular, si más de un vaso está ocluido, esto se deberá discutir en conjunto con el equipo tratante para no caer en ensañamiento terapéutico, o bien, en la omisión de un tratamiento oportuno (Figura 1). 


\section{REFERENCIAS}

1. Aguayo-Moscoso X, Mora Coello CL, Proaño-Constante MJ, ReveloOchoa EJ, Molina Vásquez PA VPJ. Accidente cerebrovascular trombótico en paciente COVID-19. Rev Científica Digit INSPILIP. 2020;4.

2. Carod-Artal FJ. Neurological complications of coronavirus and COVID-19. Rev Neurol. 2020;70:311-322.

3. Beyrouti R, Adams ME, Benjamin L, Cohen H, Farmer SF, Goh YY, et al. Characteristics of ischaemic stroke associated with COVID-19. J Neurol Neurosurg Psychiatry. 2020;91:889-891. jnnp-2020-323586.

4. Markus HS. COVID-19 and stroke: a Global World Stroke Organisation perspective. Int J Stroke. 2020;15:361-364.

5. Ng Kee Kwong KC, Mehta PR, Shukla G, Mehta AR. COVID-19, SARS and MERS: a neurological perspective. J Clin Neurosci. 2020;S09675868(20)31185-1.

6. Pesce A, Palmieri M, Armocida D, Frati A, Santoro A. Neurosurgery and Coronavirus (COVID-19) epidemic: doing our Part. Neurosurgery. 2020;87:E48-E49.

7. Rothe C, Schunk M, Sothmann P, Bretzel G, Froeschl G, Wallrauch C, et al. Transmission of 2019-nCoV Infection from an Asymptomatic Contact in Germany. N Engl J Med. 2020;382:970-971.

8. Hu YJ, Zhang J-min, Chen Z-ping. Experiences of practicing surgical neuro-oncology during the COVID-19 pandemic. J Neurooncol. 2020;148:199-200.

9. Talke PO, Sharma D, Heyer EJ, Bergese SD, Blackham KA, Stevens RD. Republished: Society for Neuroscience in Anesthesiology and Critical Care expert consensus statement: anesthetic management of endovascular treatment for acute ischemic stroke. Stroke. 2014;45:e138-150.

10. Flexman AM, Abcejo AS, Avitsian R, De Sloovere V, Highton D, Juul N. Neuroanesthesia practice during the COVID-19 pandemic: Recommendations from Society for Neuroscience in Anesthesiology and Critical Care (SNACC). J Neurosurg Anesthesiol. 2020;32:202-209.

11. Lippi G, Plebani M, Henry BM. Thrombocytopenia is associated with severe coronavirus disease 2019 (COVID-19) infections: a metaanalysis. Clin Chim Acta. 2020;506:145-148.

12. Mehta P, McAuley DF, Brown M, Sanchez E, Tattersall RS, Manson JJ. COVID-19: consider cytokine storm syndromes and immunosuppression. Lancet. 2020;395:1033-1034.

13. Carrillo-Esper R, Mejía-Terrazas GE, Acosta-Nava VM, AlcántaraMuñoz MRA, Arenas-Venegas AD, Carapia-Sadurni A, et al. Guía de recomendaciones para el manejo perioperatorio de pacientes con infección por SARS-CoV-2 (COVID-19). Rev Mex Anest. 2020;43:182-213.

14. Ozoner B, Gungor A, Hasanov T, Toktas O, Kilic T. Neurosurgery practice during coronavirus disease 2019 (COVID-19) Pandemic. World Neurosurgery. 2020;140:198-207.

15. Ti LK, Ang LS, Foong TW, Ng BSW. What we do when a COVID-19 patient needs an operation: operating room preparation and guidance. Can J Anesth. 2020;67:756-758.

16. Muhammad S, Tanikawa R, Lawton MT, Niemelä M, Hänggi D. Safety instructions for neurosurgeons during COVID-19 pandemic based on recent knowledge and experience. Neurosurgery. 2020;87:E220-E221.

17. Jenkins A. Letter: transmission of COVID-19 during neurosurgical proceduressome thoughts from the united kingdom. Neurosurgery. 2020,87:E68.

18. Yu Tung Lo, Neville Wei Yang Teo, Beng Ti Ang. Editorial. Endonasal neurosurgery during the COVID-19 pandemic: the Singapore perspective in: J Neurosurg. 2020;17:1-3.

19. Grelat M, Pommier B, Portet S, Amelot A, Barrey C, Leroy HA, et al. Patients with coronavirus 2019 (COVID-19) and surgery: guidelines and checklist proposal. World Neurosurg. 2020;139:e769-e773.

20. Sharma D, Rasmussen M, Han R, Whalin MK, Davis M, Andrew Kofke W. Anesthetic management of endovascular treatment of acute ischemic stroke during COVID-19 pandemic: consensus statement from Society for Neuroscience in Anesthesiology \& Critical Care (SNACC) of Neurological Surgeons (CNS) Cerebrovascular Section. J Neurosurg Anesthesiol. 2020;32:193-201.
21. Osorio-Santiago MA, Leticia González-Villavelázquez M, ObregónCorona A. Manejo anestésico en terapia endovascular neurológica. Reva Mex Anest. 2012;35:S143-S147.

22. Schönenberger S, Uhlmann L, Hacke W, Schieber S, Mundiyanapurath $\mathrm{S}$, Purrucker JC, et al. Effect of conscious sedation vs general anesthesia on early neurological improvement among patients with ischemic stroke undergoing endovascular thrombectomy: a randomized clinical trial. JAMA. 2016;316:1986-1996.

23. Jumaa MA, Zhang F, Ruiz-Ares G, Gelzinis T, Malik AM, Aleu A, et al. Comparison of safety and clinical and radiographic outcomes in endovascular acute stroke therapy for proximal middle cerebral artery occlusion with intubation and general anesthesia versus the nonintubated state. Stroke. 2010;41:1180-1184.

24. Rowat AM, Dennis MS, Wardlaw JM. Hypoxaemia in acute stroke is frequent and worsens outcome. Cerebrovasc Dis. 2006;21:166-172.

25. Henninger N, Bouley J, Nelligan JM, Sicard KM, Fisher M. Normobaric hyperoxia delays perfusion/diffusion mismatch evolution, reduces infarct volume, and differentially affects neuronal cell death pathways after suture middle cerebral artery occlusion in rats. J Cereb Blood Flow Metab. 2007;27:1632-1642.

26. Djurberg HG, Seed RF, Price Evans DA, Brohi FA, Pyper DL, Tjan GT, et al. Lack of effect of $\mathrm{CO}_{2}$ on cerebral arterial diameter in man. J Clin Anesth. 1998;10:646-651.

27. Solaiman O, Singh JM. Hypocapnia in aneurysmal subarachnoid hemorrhage: Incidence and association with poor clinical outcomes. J Neurosurg Anesthesiol. 2013;25:254-261.

28. Leonardi-Bee J, Bath PMW, Phillips SJ, Sandercock PAG. Blood pressure and clinical outcomes in the international stroke trial. Stroke. 2002;33:1315-1320.

29. Van der Jagt M. Fluid management of the neurological patient: a concise review. Crit Care. 2016;20:126.

30. Kirkman MA, Citerio G, Smith M. The intensive care management of acute ischemic stroke: an overview. Intensive Care Med. 2014;40:640-653.

31. Kase CS, Furlan AJ, Wechsler LR, Higashida RT, Rowley HA, Hart RG, et al. Cerebral hemorrhage after intra-arterial thrombolysis for ischemic stroke: The PROACT II trial. Neurology. 2001;57:1603-1610.

32. Nukui S, Akiyama H, Soga K, Takao N, Tsuchihashi Y, Iijima N, et al. Risk of hyperglycemia and hypoglycemia in patients with acute ischemic stroke based on continuous glucose monitoring. J Stroke Cerebrovasc Dis. 2019;28:104346.

33. Gray CS, Hildreth AJ, Sandercock PA, O’Connell JE, Johnston DE, Cartlidge NEF. Articles glucose-potassium-insulin infusions in the management of post-stroke hyperglycaemia: the UK glucose insulin in stroke trial (GIST-UK). Lancet Neurol. 2007;6:P397-406.

34. Akpinar SH, Yilmaz G. Periprocedural complications in endovascular stroke treatment. Br J Radiol. 2016;89:20150267.

35. Gill HL, Siracuse JJ, Parrack IK, Huang ZS, Meltzer AJ. Complications of the endovascular management of acute ischemic stroke. Vasc Health Risk Manag. 2014;10:675-681.

36. Elakkad A, Drocton G, Hui F. Endovascular stroke interventions: procedural complications and management. Semin Intervent Radiol. 2020;37:199-200.

37. Chen P, Xiong X-H, Chen Y, Wang K, Zhang Q-T, Zhou W. Perioperative management strategy of severe traumatic brain injury during the outbreak of COVID-19 Perioperative management strategy of severe traumatic brain injury during the outbreak of COVID-19. Chinese J Traumatol. 2020. In Press. Journal Pre Proof.

38. Sibert KS, Long JL, Haddy SM. Extubation and the risks of coughing and laryngospasm in the era of coronavirus disease-19 (COVID-19). 2020;12:e8196.

39. Chen T, Wu D, Chen H, Yan W, Yang D, Chen G, et al. Clinical characteristics of 113 deceased patients with coronavirus disease 2019: retrospective study. BMJ. 2020;26:368. 